\title{
Large Cell Neuroendocrine Carcinoma of the Colon With Carcinomatosis Peritonei
}

\author{
Jang Jin Kim ${ }^{1}$, Sung Su Park ${ }^{1}$, Taek-Gu Lee ${ }^{1}$, Ho-Chang Lee $^{2,3}$, Sang-Jeon Lee ${ }^{1,3}$ \\ ${ }^{1}$ Department of Surgery, Chungbuk National University Hospital, Cheongju; Departments of ${ }^{2}$ Pathology and ${ }^{3}$ Surgery, College of Medicine, \\ Chungbuk National University, Cheongju, Korea
}

Colorectal large-cell neuroendocrine carcinomas (NECs) are extremely rare and have very poor prognosis compared to adenocarcinomas. A 74-year-old man presented with abdominal pain, diarrhea and hematochezia. The histopathologic report of colonoscopic biopsy performed at a local clinic was a poorly differentiated carcinoma. An abdominopelvic computed scan revealed irregularly enhanced wall thickening at the sigmoid colon with regional fat stranding and lymphnode enlargement. He underwent a laparoscopic high anterior resection with selective peritonectomy for peritoneal carcinomatosis, intraoperative peritoneal irrigation chemotherapy, and early postoperative intraperitoneal chemotherapy for 5 days. The tumor had a high proliferation rate (mitotic count $>50 / 10 \mathrm{HPFs}$ and $90 \%$ of the Ki-67 index) and lymph-node metastases had occurred. On immunohistochemistry, the tumor cells expressed CD56 and synaptophysin. Large-cell NEC was confirmed. Systemic chemotherapy with cisplatin/etoposide was done. The patient is still alive after 3 years with no evidence of recurrence.

Keywords: Large-cell neuroendocrine carcinoma; Colon; Peritoneal carcinomatosis

\section{INTRODUCTION}

Neuroendocrine neoplasms (NENs) are neoplasms that have neuroendocrine differentiation and express various specific neuroendocrine markers. Most neuroendocrine tumors have a better prognosis than conventional adenocarcinomas. However, neuroendocrine carcinomas (NECs) are a highly malignant subgroup of NENs and can be classified into 2 types, small cell or large cell. Gastroenteropancreatic (GEP)-NECs have very poor survival, with a median of only 4-16 months, depending on the disease stage and the primary site [1]. Colorectal NECs seems to have an even direr prognosis than other GEP-NECs, with almost all patients dying within a year, the median overall survival time being

Received: December 27, 2017 - Accepted: February 27, 2018

Correspondence to: Sang-Jeon Lee, M.D.

Department of Surgery, College of Medicine, Chungbuk National University, 1 Chungdae-ro, Seowon-gu, Cheongju 28644, Korea

Tel: +82-43-269-6360, Fax: +82-43-266-6037

E-mail: colon@chungbuk.ac.kr

ORCID code: https://orcid.org/0000-0001-7883-5858

(C) 2018 The Korean Society of Coloproctology

This is an open-access article distributed under the terms of the Creative Commons Attribution NonCommercial License (http://creativecommons.org/licenses/by-nc/4.0) which permits unrestricted noncommercial use, distribution, and reproduction in any medium, provided the original work is properly cited. usually between 4 and 16 months [2-5]. Fortunately, NECs of the colon and the rectum are rare, representing less than $1 \%-2 \%$ of colorectal cancers $[4,6]$. Furthermore, large-cell NECs account for only about $0.25 \%$ of colorectal cancers; they are extremely rare [4], and few reports on large-cell colorectal NECs have been published. Here, we report a case of a large-cell NEC of the colon with carcinomatosis peritonei treated by using a surgical resection with early postoperative intraperitoneal chemotherapy (EPIC) and postoperative chemotherapy with cisplatin and etoposide.

\section{CASE REPORT}

A 74-year-old man presented with abdominal pain, diarrhea and hematochezia that he had been experiencing for 20 days. He had a history of hypertension and diabetes mellitus. He had undergone an appendectomy 20 years earlier. Colonoscopy in a local clinic revealed an ulcerofungating tumor within the sigmoid colon, $15-20 \mathrm{~cm}$ from the anal verge. The histopathologic report of colonoscopic biopsy was a poorly differentiated carcinoma (Fig. 1A). Abdominopelvic computed tomography (APCT) showed irregularly enhanced wall thickening at the sigmoid colon with regional fat stranding and lymph-node enlargement (Fig. 1A). Many lymph nodes at the para-aortic, interaortocaval area and at 
Volume 34, Number 4, 2018

Ann Coloproctol 2018;34(4):222-225

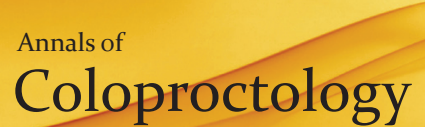

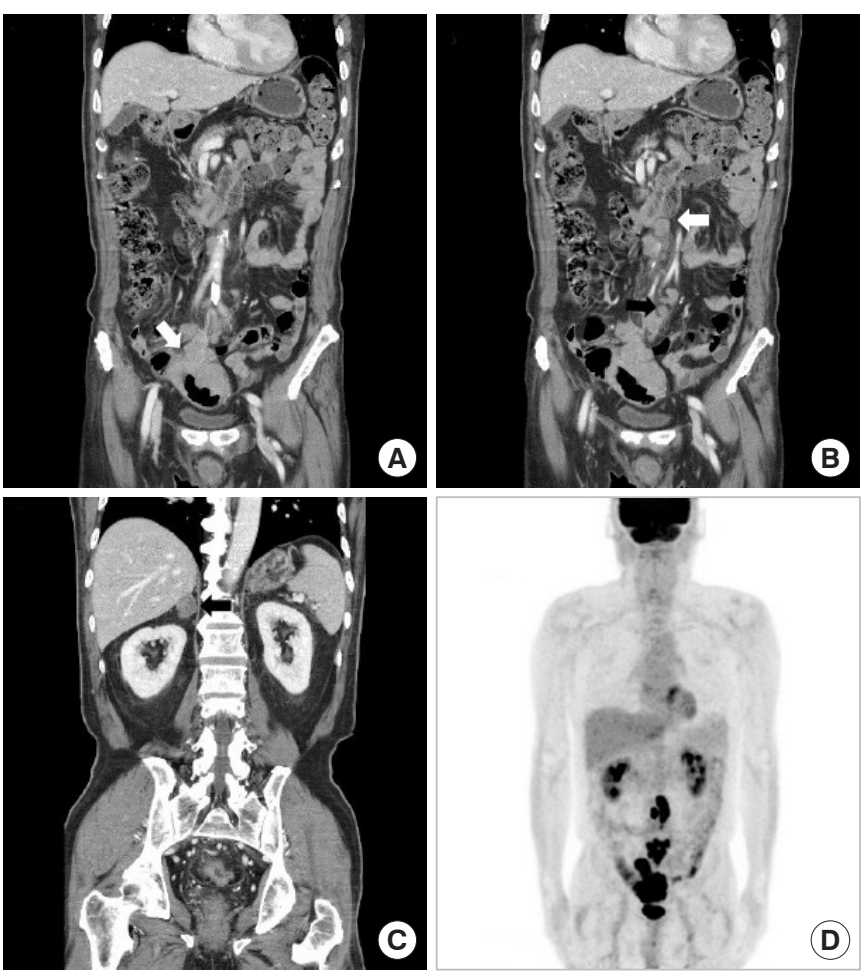

Fig. 1. Abdominopelvic computed tomography and positron emission tomography. (A) Irregularly enhanced wall thickening at the sigmoid colon with regional fat stranding (white arrow). (B) Enlarged lymph nodes at the para-aortic, interaortocaval area (white arrow) and small bowel mesentery (black arrow). (C) Heterogeneously enhanced nodular thickening at the adrenal glands. (D) Abnormal fluorodeoxyglucose uptake at the sigmoid colon with surrounding enlarged lymph nodes; left para-aortic, aortocaval, and sacral promontory; and small bowel mesentery. the small bowel mesentery were enlarged, and heterogeneously enhanced nodular thickening at both adrenal glands were also seen on APCT (Fig. 1B, C). Positron emission tomography revealed abnormal fluorodeoxyglucose uptake at the sigmoid colon with surrounding enlarged lymph nodes (SUVmax $=13.7)$ and with hypermetabolic lymph nodes at the left para-aortic, aortocaval, and sacral promontory and the small bowel mesentery (Fig. 1D). Serum carcinoembryonic antigen and carbohydrate antigen 19-9 were not elevated.

The patient underwent laparoscopic surgery. The tumor was located from the rectosigmoid junction to the distal sigmoid colon, and peritoneal seedings confined to the pelvic cavity were also found and were confirmed to be metastatically malignant by frozen biopsies. A high anterior resection with selective peritonectomy (completeness of cytoreduction score: CC-0) and intraoperative peritoneal irrigation chemotherapy (5-fluoruracil + mitomycin C) were performed. The patient also received EPIC for 5 days. The resected tumor formed an ulcerofungating mass penetrating the serosa (T4a) and measuring $6 \mathrm{~cm} \times 5 \mathrm{~cm}$ (Fig. 2A). Microscopic examination showed that the tumor consisted of highly pleomorphic cells with prominent nucleoli and a sheet-like arrangement with poor gland formation, which are unusual findings for a conventional adenocarcinoma of colorectal origin. Mitotic features were also frequently observed, which were more than 50/10 HPF. Neither lymphovascular invasion nor perineural invasion was found. Lymph-node metastases were found in 13 out of 20 dissected lymph nodes. The resection margins of both the tumor and the peritoneum were free of carcinoma. Immunohistochemistry revealed that the tumor cells were positive for neuroendocrine markers such as CD56 and synaptophysin and negative for melanoma markers including S-100 protein and HMB-45. The Ki-67 index was approximately 90\% (Fig. 2B, C).
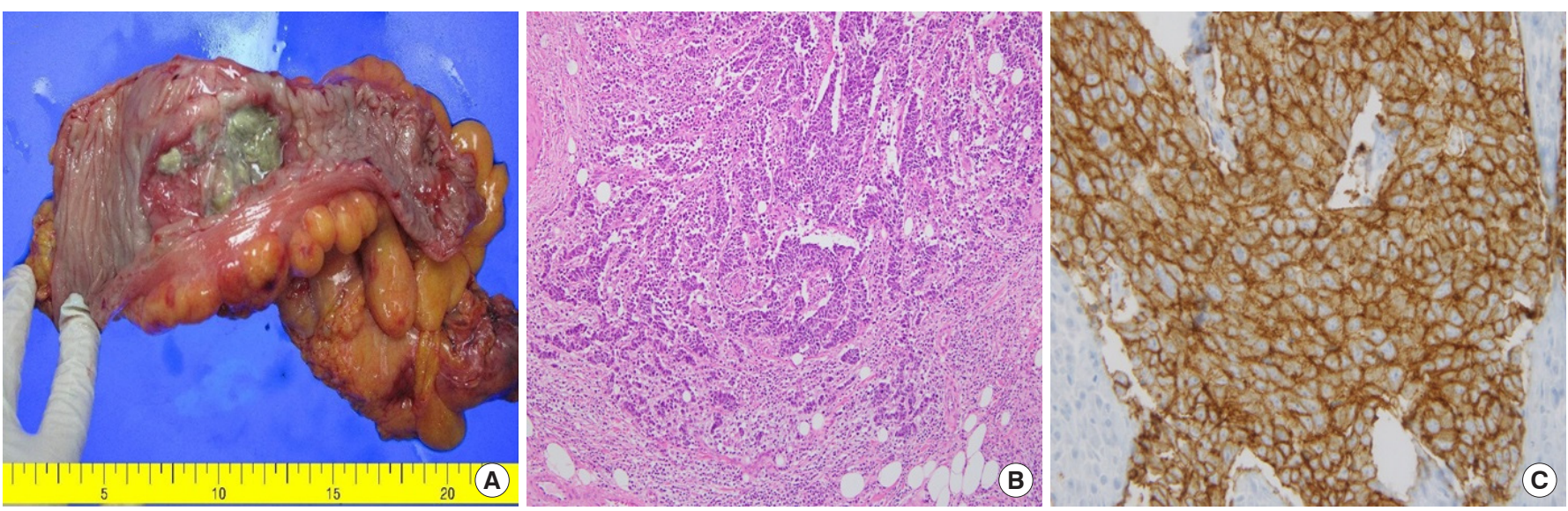

Fig. 2. (A) The resected tumor formed an ulcerofungating mass with a cross section of about $6 \mathrm{~cm} \times 5 \mathrm{~cm}$. (B) Pericolorectal adipose tissue is infiltrated by tumor cells that are highly pleomorphic and arranged in sheets. No definite formation of gland-like structures, which is very common in well-differentiated colorectal adenocarcinomas, is seen (H\&E, $\times 200)$. (C) The tumor cells are positive for CD56 and show a membrane-staining pattern $(\mathrm{CD} 56, \times 200)$. 


\section{Coloproctology Jang Jin Kim, et al.}

Four weeks after the operation, systemic chemotherapy with the cisplatin/etoposide regimen was done [7]. The patient received systemic chemotherapy for 6 months (8 cycles). After three years, he is still alive, with no evidence of recurrence.

The institutional review board of Chungbuk National University Hospital approved this case report and waived the informed consent requirement.

\section{DISCUSSION}

NENs have neuroendocrine differentiation and express various specific neuroendocrine markers such as chromogranin A ( CgA) and synaptophysin. Less specific markers include CD56 and neuron specific enolase [8]. NECs are poorly differentiated tumors with small or large cells, express neuroendocrine markers, and have a high proliferative index (grade 3 with a Ki-67 > 20\%). The classification of NENs is based on histology. Throughout the past 20 years, various classification systems have subgrouped NENs according to their differentiation and grade. The World Health Organization's 2010 Classification describes a GEP-NEC as a poorly differentiated, high-grade, malignant neoplasm composed of small cells or large-to-intermediate cells. The grading system currently used for the classification of all GEP-NENs is based on the Ki-67 proliferation index or mitotic count [9], where GEPNECs meet diagnostic criteria (Ki-67 index $>20 \%$ or mitotic count $>20 / 10 \mathrm{HPF}$ ). Tumor differentiation, mitotic rate, and Ki67 index should all be included in the pathology report [7].

Large-cell NECs may show an organoid pattern with solid nests, rosette formations or acinar structures, focal necrosis, and high mitotic rate. They have a low nucleus-to-cytoplasm ratio, nuclei with evident nucleoli and vesicular chromatin; often, they also have abundant eosinophilic cytoplasm [9]. The tumors are argyrophilic in immunohistochemical staining. Commonly, synaptophysin is diffusely positive while CgA expression is frequently negative $[1,9]$. Large-cell NECs are often similar to poorly differentiated carcinomas in microscopic figures. For the prevention of misdiagnosis, confirming the neuroendocrine markers in immunohistochemical staining is important $[10,11]$. In this case, the histopathologic report of colonoscopic biopsy performed at a local clinic was a poorly differentiated carcinoma. Colorectal NECs should be histologically distinguished from lymphomas, squamous cell carcinomas with basaloid features, malignant melanomas, and adenocarcinomas by using their immunohistochemical and morphological characteristics [12].

In this case, immunohistochemistry revealed that the tumor cells were positive for neuroendocrine markers such as CD56 and synaptophysin and negative for melanoma markers including S-100 protein and HMB-45. Clinically, colorectal NECs differ from colorectal adenocarcinomas. They are more aggressive and have frequent distant metastases and poorer survival $[6,12]$. Vascular, lymphatic and perineural invasion, as well as lymph node metastases, are usually found at diagnosis [13]. Proliferation rates are generally high, with Ki-67 values $>90 \%$ and mitotic cell counts $>$ $140 / 10 \mathrm{HPF}[11,12]$. This patient also showed a high proliferation rate (mitotic count $>50 / 10$ HPFs and Ki-67 index of 90\%).

No consensus exists for the treatment of colorectal NECs with peritoneal seedings. European Neuroendocrine Tumor Society guidelines recommend that surgery for rectal and colonic NECs be performed as for an adenocarcinoma [13]. In a retrospective study, a primary tumor resection was found to have no survival advantage, but chemotherapy improved survival in metastatic patients [14]. Bernick et al. [4] recommend cisplatin and etoposide for stage III and IV tumors. Also, the National Comprehensive Cancer Network guideline states that cisplatin/etoposide may be appropriate for patients with an atypical or poorly differentiated carcinoma [7]. Chemoradiation seems to be the most efficacious treatment for tumors with atypical histology or tumors with higher mitotic and proliferative indices. However, these recommendations are not suitable for gastrointestinal NECs.

In this case, we performed a high anterior resection and peritonectomy for the seedings within the pelvic cavity, as well as peritoneal lavage with 5 -fluorouracil/mitomycin $\mathrm{C}$. The patients also received EPIC for 5 days. The role of intraperitoneal chemotherapy in carcinomatosis peritonei is not proven yet, but some retrospective analyses showed that cytoreductive debulking with hyperthermic intraperitoneal chemotherapy may be beneficial for peritoneal carcinomatosis without extra-abdominal metastases. Four weeks after the operation, systemic chemotherapy with the cisplatin/etoposide regimen was done [7]. Colorectal NECs seem to have an even direr prognosis than other GEP-NECs, with almost all patients dying within a year, the median overall survival time usually being between 4 and 16 months [2-5]. However, our patient is still alive three years after the operation and shows no signs of recurrence.

In conclusion, to our best knowledge, this is the first report on a colorectal large-cell NEC with carcinomatosis peritonei treated as a colorectal adenocarcinoma with peritoneal seedings. This case shows that a complete surgical resection, intraperitoneal chemotherapy, early postoperative intraperitoneal chemotherapy, and systemic chemotherapy (cisplatin/etoposide) may play important roles in improving survival in patient with a colorectal large-cell NEC with carcinomatosis peritonei.

\section{CONFLICT OF INTEREST}

The authors declare no conflict of interest.

\section{REFERENCES}

1. Ilett EE, Langer SW, Olsen IH, Federspiel B, Kjær A, Knigge U. Neuroendocrine carcinomas of the gastroenteropancreatic system: a comprehensive review. Diagnostics (Basel) 2015;5:119-76.

2. Sorbye H, Welin S, Langer SW, Vestermark LW, Holt N, Osterlund $\mathrm{P}$, et al. Predictive and prognostic factors for treatment and 
survival in 305 patients with advanced gastrointestinal neuroendocrine carcinoma (WHO G3): the NORDIC NEC study. Ann Oncol 2013;24:152-60.

3. Yamaguchi T, Machida N, Morizane C, Kasuga A, Takahashi H, Sudo K, et al. Multicenter retrospective analysis of systemic chemotherapy for advanced neuroendocrine carcinoma of the digestive system. Cancer Sci 2014;105:1176-81.

4. Bernick PE, Klimstra DS, Shia J, Minsky B, Saltz L, Shi W, et al. Neuroendocrine carcinomas of the colon and rectum. Dis Colon Rectum 2004;47:163-9.

5. Patta A, Fakih M. First-line cisplatin plus etoposide in high-grade metastatic neuroendocrine tumors of colon and rectum (MCRC NET): review of 8 cases. Anticancer Res 2011;31:975-8.

6. Kang H, O’Connell JB, Leonardi MJ, Maggard MA, McGory ML, Ko CY. Rare tumors of the colon and rectum: a national review. Int J Colorectal Dis 2007;22:183-9.

7. National Comprehensive Cancer Network. NCCN clinical practice guidelines in oncology (NCCN Guidelines). Version 3 [Internet]. Fort Wathington (PA): National Comprehensive Cancer Network; 2017 [cited 2017 Dec 12]. Available from: https://www. nccn.org/professionals/physician_gls/pdf/neuroendocrine.pdf.

8. Rindi G, Wiedenmann B. Neuroendocrine neoplasms of the gut and pancreas: new insights. Nat Rev Endocrinol 2011;8:54-64.

9. Bosman FT, Carneiro F, Hruban RH, Theise ND. WHO classifi- cation of tumours of the digestive system. 4th ed. Lyon (France): International Agency for Research on Cancer (IARC); 2010.

10. Janson ET, Sorbye H, Welin S, Federspiel B, Grønbæk H, Hellman P, et al. Nordic guidelines 2014 for diagnosis and treatment of gastroenteropancreatic neuroendocrine neoplasms. Acta Oncol 2014;53:1284-97.

11. La Rosa S, Marando A, Furlan D, Sahnane N, Capella C. Colorectal poorly differentiated neuroendocrine carcinomas and mixed adenoneuroendocrine carcinomas: insights into the diagnostic immunophenotype, assessment of methylation profile, and search for prognostic markers. Am J Surg Pathol 2012;36:601-11.

12. Gaffey MJ, Mills SE, Lack EE. Neuroendocrine carcinoma of the colon and rectum. A clinicopathologic, ultrastructural, and immunohistochemical study of 24 cases. Am J Surg Pathol 1990;14:101023.

13. Strosberg JR, Cheema A, Weber JM, Ghayouri M, Han G, Hodul PJ, et al. Relapse-free survival in patients with nonmetastatic, surgically resected pancreatic neuroendocrine tumors: an analysis of the AJCC and ENETS staging classifications. Ann Surg 2012;256: 321-5.

14. Smith JD, Reidy DL, Goodman KA, Shia J, Nash GM. A retrospective review of 126 high-grade neuroendocrine carcinomas of the colon and rectum. Ann Surg Oncol 2014;21:2956-62. 